\title{
Relaxation, closing probabilities and transition from oscillatory to chaotic attractors in asymmetric neural networks
}

\author{
Ugo Bastolla $^{1}$ and Giorgio Parisi ${ }^{2}$
}

June 5,2022

\author{
${ }^{1}$ HLRZ, Forschungszentrum Jülich, D-52425 Jülich Germany \\ ${ }^{2}$ Dipartimento di Fisica, Università "La Sapienza", P.le Aldo Moro 2, I-00185 Roma Italy \\ Keywords: Disordered Systems, Attractor Neural Networks
}

\begin{abstract}
Attractors in asymmetric neural networks with deterministic parallel dynamics present a "chaotic" regime at symmetry $\eta \leq 0.5$ where the average length of the cycles increases exponentially with system size, and an oscillatory regime at high symmetry, where the average length of the cycles is 2 [10. We show, both with analytic arguments and numerically, that there is a sharp transition, at a critical symmetry $\eta_{c}=0.33$, between a phase where the typical cycles have length 2 and basins of attraction of vanishing weight and a phase where the typical cycles are exponentially long with system size, and the weights of their attraction basins are distributed as in a Random Map with reversal symmetry. The time-scale after which cycles are reached grows exponentially with system size $N$, and the exponent vanishes in the symmetric limit, where $T \propto N^{2 / 3}$. The transition can be related to the dynamics of the infinite system (where cycles are never reached), using the closing probabilities as a tool.

We also study the relaxation of the function $E(t)=-1 / N \sum_{i}\left|h_{i}(t)\right|$, where $h_{i}$ is the local field experienced by the neuron $i$. In the symmetric system, it plays the role of a Ljapunov function which drives the system towards its minima through steepest descent. This interpretation survives, even if only on the average, also for small asymmetry. This acts like an effective temperature: the larger is the asymmetry, the faster is the relaxation, and the higher is the asymptotic value reached. $E$ reachs very deep minima in the fixed points of the dynamics, which are reached with vanishing probability, and attains a larger value on the typical attractors, which are cycles of length 2 .
\end{abstract}

\section{Introduction}

The dynamics of Attractor Neural Networks with randomly distributed synaptic couplings has been investigated in several works in recent years, with particular attention to the 
effects of the asymmetry of the synaptic couplings [1, 2, 3, 4, 5, 7, 8, 9, 10, 11, 12, 13, 14. This generalization is interesting not only because in the brain synapses are not symmetric, but also because it introduces new complex features in the dynamics of such systems. At symmetry higher than a critical value the system does never completely lose memory of its initial state [6, 9, 12, while at zero symmetry the dynamics have the essential characteristics of chaos in continuous systems even if the model has a finite state space [13] (in this case it has been shown analytically that chaos is suppressed by strong enough thermic noise [15]).

At low symmetry the average length of the attractors increases exponentially with the size of the system, while for high symmetry the typical attractors are cycles of length 2 . This kind of transition takes place also in other discrete disordered dynamical systems [16]. The model that we consider here is very simple, and a detailed study of this transition is possible. Moreover, the study of asymmetry is interesting in the context of neural networks, both because it drops the unrealistic requirement of symmetric synaptic couplings formulated in the classical Hopfield model [18] and because the existence of chaotic attractors can be a way to distinguish between a network converging to a regular attractor since it has remembered a pattern and a network which is in a confused state [1]. On the other hand, the model that we study can also be seen as a modification of the mean field model of Spin Glass proposed by Sherrington and Kirkpatrick [20], at temperature $T=0$ (if the couplings are not symmetric the system is not Hamiltonian, but also in the case of symmetric couplings the Hamiltonian of the system is different from the SK Hamiltonian due to the parallel dynamics, see below).

The model that we consider consists in $N$ Ising neurons $\sigma_{i}= \pm 1$ which are updated simultaneously according to deterministic rules:

$$
\sigma_{i}(t+1)=\operatorname{sign}\left(\sum_{j} J_{i j} \sigma_{j}(t)\right) .
$$

The $J_{i j}$ (synaptic couplings) are quenched random variables chosen from a distribution with average value zero and variance $1 / N$. The exact form of the distribution is irrelevant in the infinite size limit, so we chose a two-valued distribution which is easiest to implement numerically [9]. The degree of symmetry of the synaptic couplings is parameterized by $\eta$, which measures the correlation between $J_{i j}$ and $J_{j i}$ :

$$
\eta=\frac{\left\langle J_{i j} J_{j i}\right\rangle}{\left\langle J_{i j}^{2}\right\rangle} .
$$

If the limit $N \rightarrow \infty$ is taken before the limit $t \rightarrow \infty$, it is possible to apply a MonteCarlo method that reproduces exactly the dynamics of a single spin in the infinite system [17]. This approach [12] and previous numerical works [6, 9] show the presence of a phase transition at $\eta_{R M}=0.825$. At symmetry larger than $\eta_{R M}$ a system that was initially in a magnetized state does never forget the initial configuration, and the remanent magnetization, $m_{\infty}=\lim _{l \rightarrow \infty} C(0, l)$, where $C$ is the correlation function, is different from zero. At low symmetry the initial condition is completely forgotten.

If the limit $t \rightarrow \infty$ is taken in a finite system, a different situation is found. Since configuration space is finite and the dynamics is deterministic, after a transient time the motion takes place on periodic orbits. The length of such orbits, the size of their attraction basins and the number of orbits are random variables, depending on the realization of the 
couplings $J_{i j}$. We are interested in their scaling behavior as a function of system size, $N$. We can wonder what is the relation of this situation with the dynamics of an infinite system, where attractors do not exist. We will show that the properties of the attractors can be deduced from the statistical properties of the correlation function in the infinite system.

If the synaptic couplings are symmetric $\left(i . e ., J_{i j}=J_{j i}\right)$, one can define [19] the function

$$
E(t)=-\sum_{i, j} \sigma_{i}(t+1) J_{i j} \sigma_{j}(t)=-\sum_{i}\left|\sum_{j} J_{i j} \sigma_{j}(t)\right|,
$$

which is a non-increasing function of time. Since $E(t)$ attains a constant value only on fixed points and on cycles of length 2, these are the only attractors of the system. On the fixed points the function $E$ coincides with the Hamiltonian of the SK model of Spin Glass [20], $H=-\sum_{i j} J_{i j} \sigma_{i} \sigma_{j}$, thus the fixed points of the symmetric networks are also metastable states for the SK model. The typical attractors are nevertheless cycles of length 2: nearly every initial point (in the limit $N \rightarrow \infty$ ) converges to a cycle of length 2. For symmetry reasons, the value of $H$ is in average zero in such cycles, but $E$ has a low value. We observed that the lowest values of $E$ are attained on the fixed points, which are reached with vanishing probability. Thus, under the point of view of relaxation, cycles of length 2 act as traps.

Let us summarize our results about non-symmetric synaptic couplings. In this case, $E(t)$ may increase and it is not anymore a Ljapunov function. Nevertheless, we saw that its average value is a non-increasing function also for asymmetric couplings. Cycles of every length may exist, but, if the asymmetry is small, the typical cycles are still cycles of length 2. Each of them has an attraction basin of vanishing weight (so that the average weight of the attraction basins is zero), but the sum of such weights tends to 1 in the infinite size limit. This situation persists up to $\eta_{c} \approx 0.33$, where the sum of the weights of cycles of length 2 suddenly drops to 0 in the infinite size limit. The typical attractors are now very long cycles, whose length increases exponentially with system size (chaotic phase). The number of such cycles is much smaller than the number of cycles of length 1 , but the average weight of their attraction basins is finite, like in the Random Map model [24]. Indeed, we argue that the distribution of the weights is the same as in a Random Map model with reversal symmetry, as in the limit case $\eta=0$ [21] $]_{\text {. }}$.

Between $\eta=0.5$ and $\eta=0.33$ long cycles are still a negligible portion of phase space, but the average length is dominated by the tails of the distribution, and increases exponentially with system size, in agreement with the results of Nützel [10]. However, we prefer to place the transition between the two regimes at $\eta_{c}=0.33$, where the nature of the typical attractors abruptly changes. We also measured the typical transient time (the time necessary to reach a cycle). It appears from our data, in contrast with previous numerical results, that the transient time increases exponentially with system size for $\eta<1$, and as $N^{2 / 3}$ for $\eta=1$. For $\eta \approx 1$ there are two regimes: a power law at small $N$ (roughly with the same exponent $2 / 3$ as in the case $\eta=1$ ) and an exponential increase for larger systems, with an exponent vanishing in the limit $\eta \rightarrow 1$. This behavior is in agreement with our theoretical expectations

\footnotetext{
${ }^{1}$ Another important model with symmetric couplings is the Hopfield model [18], the prototype of Attractors Neural Networks, where the $J_{i j}$ are given by the Hebbs rule, $J_{i j}=\sum_{\mu=1}^{p} \xi_{i}^{\mu} \xi_{j}^{\mu}$.

${ }^{2}$ The case $\eta=0$ is in some sense peculiar, since the average number of attractors increases linearly with system size [25], as in a Random Map and in contrast with the case $\eta \neq 0$, where the number of fixed points increases exponentially with $\mathrm{N}[5]$.
} 
(see below).

The above observations hold for $\eta>0$. The case $\eta<0$, which corresponds to antisymmetric couplings, is related in a simple way to the symmetric case in the infinite size limit, as we shall see. In this case the phase space is dominated by cycles of length 4 for $\eta \leq-0.33$, while exponentially long cycles prevail for larger values of $\eta$. The transition point is $\eta_{c}=-0.33$.

We study, numerically and in part analytically, the closing probabilities, that express the probability that a trajectory not yet closed closes on a cycle of length $l$ after a time $t+l$. The closing probabilities are nothing but the tail of the distribution of the correlation function $q(t, t+l)$, thus they establish a link between the properties of the infinite system and the properties of the attractors [22]. In this framework, the transition can be understood in this way: since the distribution of $q(t, t+2)$ is peaked around a much larger value than for any other value of $l$, the closing probability on cycles of length 2 is much larger than for any other cycle, for a factor exponentially large with system size. This effect decreases as $\eta$ decreases, and, at a critical parameter, it is not enough to balance the large number of possible long cycles, which then prevail.

The paper is organized as follows: in section 2 we study analytically the properties of the attractors under some hypothesis on the closing probabilities. We derive a condition on the exponent of the closing probability that corresponds to the dynamical transition. We argue that the distribution of the weights of the attraction basins is the same as for a Random Map with reversal symmetry in the whole chaotic phase, while the typical weights tend to zero in the oscillatory phase. Thus also this quantity has a discontinuity at the transition. In section 3 we present our numerical results, reporting the properties of the attractors, the relaxation of $\overline{E(t)}$, the distribution of the correlation functions and the closing probabilities. In the final discussion we point out possible extensions of this study.

\section{Closing probabilities and attractors}

The natural distance in the configuration space of the system is the Hamming distance, that measures the number of elements in a different state in two configurations. An equivalent information is given by the correlation between configurations, or overlap. Let us consider the overlap between two configurations at different times along the same trajectory:

$$
q(t, t+l)=\frac{1}{N} \sum_{i} \sigma_{i}(t) \sigma_{i}(t+l) .
$$

This is a random variable, depending a) on the quenched disorder (dynamical rules), and

b) on the randomly chosen initial point. Knowing its distribution, we can reconstruct all the properties of the attractors. In particular, we have to compute the probability that $q(t, t+l)$ is equal either to 1 or to -1 . The first case corresponds to a trajectory that, after a transient time $t$, enters a limit cycle of length $l$. The case $q(t, t+l)=-1$ corresponds to a trajectory that, after a transient. time $t$, enters a cycle of length $2 l$. In fact, if $C(t)$ has been reversed after a time $l$, also $C(t+l)$ shall be reversed after a time $l$, since the map (1) commutes with the reversal operator $\mathcal{R}$ defined by $\mathcal{R}\left\{\sigma_{1}, \cdots \sigma_{N}\right\}=\left\{-\sigma_{1}, \cdots-\sigma_{N}\right\}$, thus $C(t+2 l)$ will be equal to $C(t)$. 
We are interested in the first time when a trajectory "closes" visiting a configuration already attained. Thus, we have to impose the condition that no configuration has yet been repeated before the time $t+l$. The closing probabilities are then conditional probabilities:

$$
\begin{aligned}
& \pi_{N}^{+}\left(t, t^{\prime}\right)=\operatorname{Pr}\left\{q\left(t, t^{\prime}\right)=1 \mid A_{t^{\prime}}\right\}, \\
& \pi_{N}^{-}\left(t, t^{\prime}\right)=\operatorname{Pr}\left\{q\left(t, t^{\prime}\right)=-1 \mid A_{t^{\prime}}\right\},
\end{aligned}
$$

where the symbol $A\left(t^{\prime}\right)$ represents the condition that it never happened, before time $t^{\prime}$, either $q=1$ or $q=-1$.

\subsection{The overlap in an infinite system}

Taking this opening condition into account reconciles the apparent discrepancy between the behavior of a finite system [11] and of an infinite one [12]. In an infinite system, the average overlap after two time-steps, $Q(t, t+2)=\overline{q(t, t+2)}$, tends, as $t \rightarrow \infty$, to an asymptotic value that is an analytic function of $\eta$. In contrast, in a finite system, if time is long enough a limit cycle is reached. Then $Q(t, t+2)$ tends to 1 with probability 1 in the oscillatory phase while it is less than 1 in the chaotic phase.

Because of the opening condition we can use, as a first approximation, the properties of the correlation function in an infinite system to derive the properties of the attractors in a finite system. We list here some properties of the conditional distribution of $q(t, t+l)$ which we expect to hold if the limit $N \rightarrow \infty$ is taken with $t$ and $l$ fixed. In what follows, the opening condition is always assumed to hold.

We consider first $\eta \geq 0$. Qualitatively, we expect that the behavior of the overlap at $-\eta$ is related in a simple way to the one at $\eta$. A simple argument runs like this: all the couplings where $J_{i j} J_{j i}$ is positive tend to align $\sigma_{i}(t)$ and $\sigma_{i}(t+l)$, so that for $\eta>0$ there is an effective ferromagnetic interaction between the time slice at time $t$ and the time slice at time $t+l$, and an effective antiferromagnetic interaction for $\eta<0$. These interactions have a different sign, but the same strength. They determine the main features of the overlap distribution: for instance, the variance and the non-vanishing value of $Q(t, t+l)$ (for even $l$; for odd $l$ the distribution is symmetric)

1. The overlap distribution depends exponentially on system size:

$$
\operatorname{Pr}\{q(t, t+l)=q\} \approx \frac{A_{t, t+l}(q ; \eta)}{\sqrt{N}} \exp \left(-N \alpha_{t, t+l}(q ; \eta)\right) .
$$

This is a kind of finite entropy statement, and it can be understood as follows: the overlap is the average of $N$ terms. They are not independent, but their correlations are small enough so that the variance of the overlap is of order $1 / N$. Thus, in analogy with the Shannon-Mc Millan theorem, we expect (6) $)^{\text {. }}$.

\footnotetext{
${ }^{3}$ Here, as it is usual in the theory of disordered systems, the over-line denotes the average respect to the disorder, while the angular brackets denote an average over the initial configurations that in this case is not needed [23].

${ }^{4}$ Another way to see this equation is to compute the probability of $q(t, t+l)$ with the dynamical functional integral.
} 
2. Weak time translation invariance: in the limit $t \rightarrow \infty$, the overlap $q(t, t+l)$ converges to a well-defined limit distribution. We call $\overline{\alpha_{l}}(\eta)$ the limit value of $\alpha_{t, t+l}(q=1, \eta)$.

3. Symmetry: for $l$ odd, the distribution of $q(t, t+l)$ is symmetric around $q=0$. This can be easily proved using the inversion symmetry $J \rightarrow-J$ of the distribution of the synaptic couplings. It implies that $Q(t, t+l)=0$ for $l$ odd, and, more in general, $\alpha_{t, t+l}(q ; \eta)=\alpha_{t, t+l}(-q ; \eta)$.

The variance of the distribution is larger than $1 / N$ (the value for a binomial distribution), since there is a positive correlation between $\sigma_{i}(t) \sigma_{i}\left(t^{\prime}\right)$ and $\sigma_{j}(t) \sigma_{j}\left(t^{\prime}\right)$ if $J_{i j}$ and $J_{j i}$ have the same sign. The closing probability is accordingly larger than $2 / 2^{N}$. Both the variance and the closing probability decrease with $l$ and increase with $\eta$.

4. For $l$ even, the most probable overlap, $Q(t, t+l)$, is positive and decreases with $l$ (correlations decay in time). In the phase where there is not remanent magnetization $(\eta<0.825)$ the limit value is zero, and $q(t, t+l)$ reaches asymptotically a symmetric distribution (the limit of large $t$ having been taken in advance). The closing probability consequently decreases with $l$ and increases with $\eta$.

5. The limit distribution reached in the limit $l \rightarrow \infty$ is found numerically to be the same both for even and odd $l$. We denote the exponent of the closing probability by the symbol $\alpha_{t, \infty}(\eta)$ (since the limit distribution is symmetric, the exponent is the same both for closing with $q=1$ and for closing with $q=-1$ ).

As a consequence, the closing probability has a maximum for $l=2$ at fixed $t$, and it is exponentially larger than for any other value of $l$. Thus cycles of length 2 are found with the highest frequency.

In antisymmetric networks $(\eta<0)$ the situation is slightly more complicate: we have to distinguish $l$ odd, $l=4 m$ and $l=2(2 m+1)$.

- For odd $l$ the average overlap is zero and the variance is smaller than in a binomial distribution, since the correlation between $\sigma_{i}(t) \sigma_{i}\left(t^{\prime}\right)$ and $\sigma_{j}(t) \sigma_{j}\left(t^{\prime}\right)$ is negative if $J_{i j}$ and $J_{j i}$ have opposite sign. Thus the closing probability is smaller than in the case of a binomial distribution and increases as $l$ increases. Accordingly, cycles of odd length are very rare in antisymmetric networks.

- If $l=2(2 m+1)(l=2,6,10, \cdots)$ there is an effective antiferromagnetic interaction between times slices $t$ and $t+l$. Thus $Q(t, t+l)$ is negative and the trajectories close preferentially with $q(t, t+l)=-1$ producing cycles of length $2 l$.

- If $l=4 m$ the effective interaction is ferromagnetic, and the behavior is the same as for positive $\eta$.

In the case of negative $\eta$, we expect $\pi_{N}^{-}(t, t+l ;-\eta) \approx \pi_{N}^{-}(t, t+l ; \eta)$ for even $\eta$, asymptotically in $N$. Thus the dominant closing probability is $\pi_{N}^{-}(t, t+2)$, and the most frequent cycles are cycles of length 4 . Since the transition regarding the attractors is governed by the comparison between the closing probability with $l=2$ and that with $l=\infty$ (see below), the 
transition for negative $\eta$ should take place at $\eta_{c}^{\prime}=-\eta_{c}$. Simulations confirm very well these arguments, so that in most of what follows we limit our study to the case $\eta>0$.

In finite systems the situation is more complicate, and some of these properties do not hold when the times $t$ and $l$ are large respect to system size. For instance, in this case $q(t, t+l)$ does not reach an asymptotic distribution in the limit $t \rightarrow \infty$ : it is easy to see that its average value has to decrease at large $t$ as a consequence of the opening condition. In fact, $\tilde{\pi}_{N}\left(t^{\prime}\right)=\sum_{t} \pi_{N}\left(t, t^{\prime}\right)$ (integrated closing probability) can not exceed 1, since it represents the probability that a trajectory closes at time $t^{\prime}$. Thus $\pi_{N}(t, t+l)$ must finally decrease with $t$ and the conditional distribution of the overlap $q(t, t+l)$ can not become really stationary in $t$. However, as $N$ increases this effect becomes weaker and weaker.

We are interested in the closure of the cycles, which takes place at a time-scale $\tau$ exponentially increasing with $N$, and it is not a priori clear whether we can neglect these finite size effects or not. However, the predictions that can be drawn from this simplified description capture the essential features observed in the simulations. We derive in the following these predictions.

\subsection{Cycles of length 2}

We compute now the probability that a trajectory closes on a cycle of length $l=2$. Let us consider $\eta>0$. We want to show that, if $\bar{\alpha}_{2}(1 ; \eta)<\frac{1}{2} \bar{\alpha}_{\infty}(1 ; \eta)$, then all trajectories reach attractors of length 2 with probability 1 as $N \rightarrow \infty$. We call this situation the oscillatory phase.

It is very easy to go from the closing probabilities to the probability distribution of cycle lengths. The probability that the trajectory has not closed up to time $t$ is given by

$$
P_{N}(t) \approx \exp \left(-\sum_{t^{\prime}=1}^{t} \sum_{l=1}^{t^{\prime}} \pi_{N}\left(t^{\prime}-l, t^{\prime}\right)\right),
$$

so the time-scale with which this quantities decays gives the time-scale of typical closing events.

We introduce the function $f_{N}(t)$, which expresses the ratio between the probability that a cycle of length $l \neq 2$ is reached at time $t$ and the probability that a cycle of length 2 is reached at the same time:

$$
f_{N}(t ; \eta)=\frac{\sum_{l \neq 2}^{t} \pi_{N}(t-l, t)}{\pi_{N}(t-2, t)} .
$$

In the above hypothesis, this is an increasing function of $t$ when $t$ is large and the overlap distributions have reached the asymptotic value. The probability that a cycle of length 2 is reached at time $t$, under the condition that a cycle was reached, is given by $\left(1+f_{N}(t)\right)^{-1}$. This is very high for $t$ small and decreases with time, since more possible cycles enter the game. Let us compute this quantity at the time scale $\tau_{2}=e^{N \alpha_{2}}$ :

$$
f_{N}\left(\tau_{2} ; \eta\right) \approx \exp \left(N\left(2 \alpha_{2}-\alpha_{\infty}\right)\right)
$$

where $\alpha_{2}$ is the exponent of the asymptotic closing probability for cycles of length 2 . Two different situations occur: 
- $\alpha_{2}<\alpha_{\infty} / 2$ : oscillatory phase. $f_{N}(t)$ is exponentially small up to times of order $\tau_{2}=e^{N \alpha_{2}}$. But at such times the probability that a trajectory is not yet closed, $P_{N}(t)$, is very small: $P_{N}(t) \leq \exp \left(-t / \tau_{2}\right)$. Thus, for very large sizes $N$, almost all the cycles close before this time, and they are cycles of length 2 .

- $\alpha_{2}>\alpha_{\infty} / 2$ : chaotic phase. In this case, the time scale at which most of the trajectories are close is proportional to $\tau_{\infty}=e^{\alpha_{\infty} / 2}$, since it holds $P_{N}(t) \leq \exp \left(-\left(t / \tau_{\infty}\right)^{2}\right)$. At this time, $f_{N}\left(\tau_{\infty}\right)$ is already very small and the probability that at least a cycle of length 2 is reached is vanishingly small with $N$.

Since $\alpha_{2}(\eta)$ tends to zero for $\eta \rightarrow 1$ (as we noted above, in this case the most probable value of the overlap is $Q(t, t+2)=1$ and the distribution is not exponential), while $\alpha_{\infty}$ does not vanish in this limit (actually, for $\eta=1$ the value of $\alpha_{\infty}$ is infinite, since only cycles of length 1 and 2 are found), and for $\eta=0$ the exponents $\alpha_{2}$ and $\alpha_{\infty}$ are roughly equal, it must exist a critical value of $\eta$ at which the condition $\alpha_{\infty}=2 \alpha_{2}$ is fulfilled.

\subsection{Transient times}

According to the above argument, the typical closing times are given by

$$
\operatorname{Tr} \propto \exp \left(N \min \left(\alpha_{2}, \alpha_{\infty} / 2\right)\right),
$$

and the argument predicts that transient time increases exponentially with system size, with an exponent that vanishes in the limit $\eta \rightarrow 1$. Close to this value, very large systems are needed in order to distinguish this behavior from a stretched exponential or a power law. For $\eta=1$, the most likely value of $q(t, t+2)$ is $q=1$ and the exponent $\alpha_{2}$ vanishes. Thus the closing probability has to be computed before $q(t, t+2)$ reaches its stationary distribution. In the Gaussian approximation,

$$
\pi_{N}(t, t+2 ; \eta=1) \propto \frac{1}{\sqrt{N}} \exp \left(-\frac{\left(1-Q_{2}(t ; \eta=1)\right)^{2}}{2 N V_{2}(t)}\right) \approx \frac{1}{\sqrt{N}} \exp \left(-\frac{\left(1-Q_{2}(t ; \eta=1)\right)}{2 N}\right)
$$

since, for $Q_{2}$ close to 1 , the variance goes to zero as $V_{2} \propto 1-Q_{2}$. It is known from previous numerical studies 23] that $1-Q_{2}(t, \eta=1) \propto t^{3 / 2}$. Thus the time-scale at which trajectories close for $\eta=1$ is a power-law of system size:

$$
\operatorname{Tr}(\eta=1) \propto N^{2 / 3}
$$

This prediction is in good agreement with the numerical results in Nützel [10] and in the present work. When $\eta$ is close to 1 , there are two regimes: at small $N$, the trajectories close when the overlap distribution is not yet stationary. Since $Q(t, t+2)$ tends to its stationary value $Q_{2}^{*}(\eta)$ as $t^{-2 / 3}$ independent of $\eta$ [9, 12, 10], we expect and observe also in this case that $\operatorname{Tr} \propto N^{2 / 3}$. At larger size the overlap becomes stationary by the time when cycles close, and the exponential dependence shows up, with an exponent that, according to the Gaussian approximation, is given by 


$$
\alpha_{2}(\eta) \propto \frac{1-Q_{2}^{*}(\eta)}{2} .
$$

Numerically, it is found $\alpha_{2}(\eta) \propto(1-\eta)^{2}$.

\subsection{Average length of the cycles}

At the time $t \propto \exp \left(N \alpha_{2}\right)$ when typical cycles of length 2 close the probability that a long cycle close goes as $\exp \left(\left(\alpha_{2}-\alpha_{\infty}\right) N\right)$ and the average length of cycles longer than 2 grows as most as $\exp \left(\left(3 \alpha_{2}-\alpha_{\infty}\right) N\right)$. Thus, for $\alpha_{2}<\alpha_{\infty} / 3$, the typical length of the cycles and the average length coincide, and they are equal to 2 . At lower symmetry very large cycles appear with a probability that, though vanishing, is large enough to let them dominate the average length. At this point the average length of the cycles increases exponentially with system size. This change takes place inside the oscillatory phase, in which the typical cycles have length 2 . We prefer to use the probability $P(2)$ to find a cycle of length 2 as the order parameter to describe the transition, instead of using the logarithm of the average length of the cycles, which is dominated by the tails of the distribution.

Our simulations show that the transition defined by $P(2)$ happens at $\eta_{c} \approx 0.33$, a value much smaller than the threshold where the remanent magnetization vanishes, $\eta_{R M}=0.825$, and also smaller than the threshold at which the average length of the cycles starts to increase exponentially, $\eta_{L}=0.50$ [10].

\subsection{Weights of the attraction basins}

Another quantity that can be used as an order parameter is the average weight of the attraction basins. The weight $W_{\alpha}$ of the attraction basin of cycle $\alpha$ represents the probability that a random chosen configuration reaches ultimately this cycle. The $\left\{W_{\alpha}\right\}$ 's are random variables, depending on the realization of the couplings. It is convenient to define the moments of their distribution [26]:

$$
\overline{Y_{n}}=\sum_{\alpha} \overline{W_{\alpha}^{n}} .
$$

$Y_{1}$ is equal to 1 because of the normalization. The average weight of the attraction basins, $\overline{Y_{2}}$, is equal to the probability that two randomly chosen trajectories reach the same attractor [26]. Let us evaluate such a probability. We define $\pi_{N}^{(2)}\left(t, t^{\prime}\right)$ as the probability that the configuration at time $t$ on the first trajectory is equal to the configuration at time $t^{\prime}$ on the second trajectory, given that the initial configurations are chosen at random. This is, asymptotically in $t$, equal to $\exp \left(-N \alpha_{\infty}\right)$, if the remanent magnetization is zero, or smaller, otherwise. In fact, we expect that the overlap of two independent trajectories is asymptotically equal to the overlap between configurations at long time distance along the same trajectories, if the dynamics looses memory of the initial state (this requires that the remanent magnetization vanishes), and is smaller in the case in which the memory of the initial state is not lost. Predictions based on this expectation are well satisfied in other disordered dynamical systems like Kauffman networks [22] and discretized chaotic maps [27. 
Thus we expect that the time when two trajectories in the same attraction basin eventually meet grows as $\exp \left(-N \alpha_{\infty} / 2\right)$. In the oscillatory phase this is much larger than the time scale over which a trajectory reaches a cycle, $\exp \left(-N \alpha_{2}\right)$, so that we expect that the probability that two trajectories meet before reaching their limit cycles tends to zero. This is confirmed by the simulations.

In the chaotic phase the two time-scales are equal, and we expect non-vanishing $\overline{Y_{n}}$. Moreover, we expect that their values coincide with the moments of the weights of the attraction basins of a Random Map model [24], endowed with reversal symmetry, like in the previously studied case $\eta=0$ 21]. The computation follows exactly the same steps as in that case: in fact, the Random Map distribution follows only from the facts that the closing probabilities $\pi_{N}(t, t+l)$ tend to an asymptotic limit independent on both $t$ and $l$, and that the "transient" regime ( small $l$ ) does not contribute significantly to the closure of the cycles. The result of the computation is [21]:

$$
\overline{Y_{n+1}}=\frac{1}{2} \frac{(n !)^{2}}{(2 n+1) !}\left(4^{n}+2^{n}\right) .
$$

For instance, $\overline{Y_{2}}=1 / 2, \overline{Y_{3}}=1 / 3, \overline{Y_{4}}=9 / 35 \ldots$ Our numerical results are consistent with this prediction (see Fig. 1), although, for $\eta>0.1, \overline{Y_{2}}$ is still far from the asymptotic regime for all the systems that we could simulate.

\subsection{Average number of cycles}

We conclude the discussion of the properties of the attractors showing that the closing probabilities allow to compute the average number of cycles of length $l$ through the relation

$$
\overline{N_{c}}(l)=2^{N}\left(\frac{1}{l} \pi_{N}^{+}(0, l)+\frac{2}{l} \pi_{N}^{-}(0, l / 2)\right)
$$

(the last term is present only for even $l$ ). Thus the number of cycles varies exponentially with $N$. For odd $l$ and $\eta>0, \alpha_{0 l}(q=1)$ is smaller than $\ln 2$ (in fact, there is a positive correlation between $\sigma_{i}(0) \sigma_{i}(l)$ and $\sigma_{j}(0) \sigma_{j}(l)$ if $J_{i j}$ and $J_{j i}$ have the same sign. The correlation change of sign in case of negative $\eta$, and the number of odd cycles becomes exponentially small). For even $l=2 m, \alpha_{0 l}(1)$ is also smaller than $\ln 2$ (in fact, the average value of $\sigma_{i}(0) \sigma_{i}(l)$ is different from zero). The two cases are related: for instance, the number of cycles of length 2 increase as the square of the number of cycles of length 1 [5]. With our notation, this relation reads $\alpha_{01}-\alpha_{02}=\ln 2$.

For large $l$, no matter if even or odd, if the remanent magnetization is equal to zero, the distribution of $q(0, l)$ tends to a binomial distribution with average value $1 / 2$. Thus the average number of cycles of length $l$ tends to 1 for large $l$. A funny consequence of a non-vanishing remanent magnetization is that in this case the number of cycles of length $l$ increases exponentially with a non-vanishing exponent for every value of $l$.

\section{$3 \quad$ Numerical results}



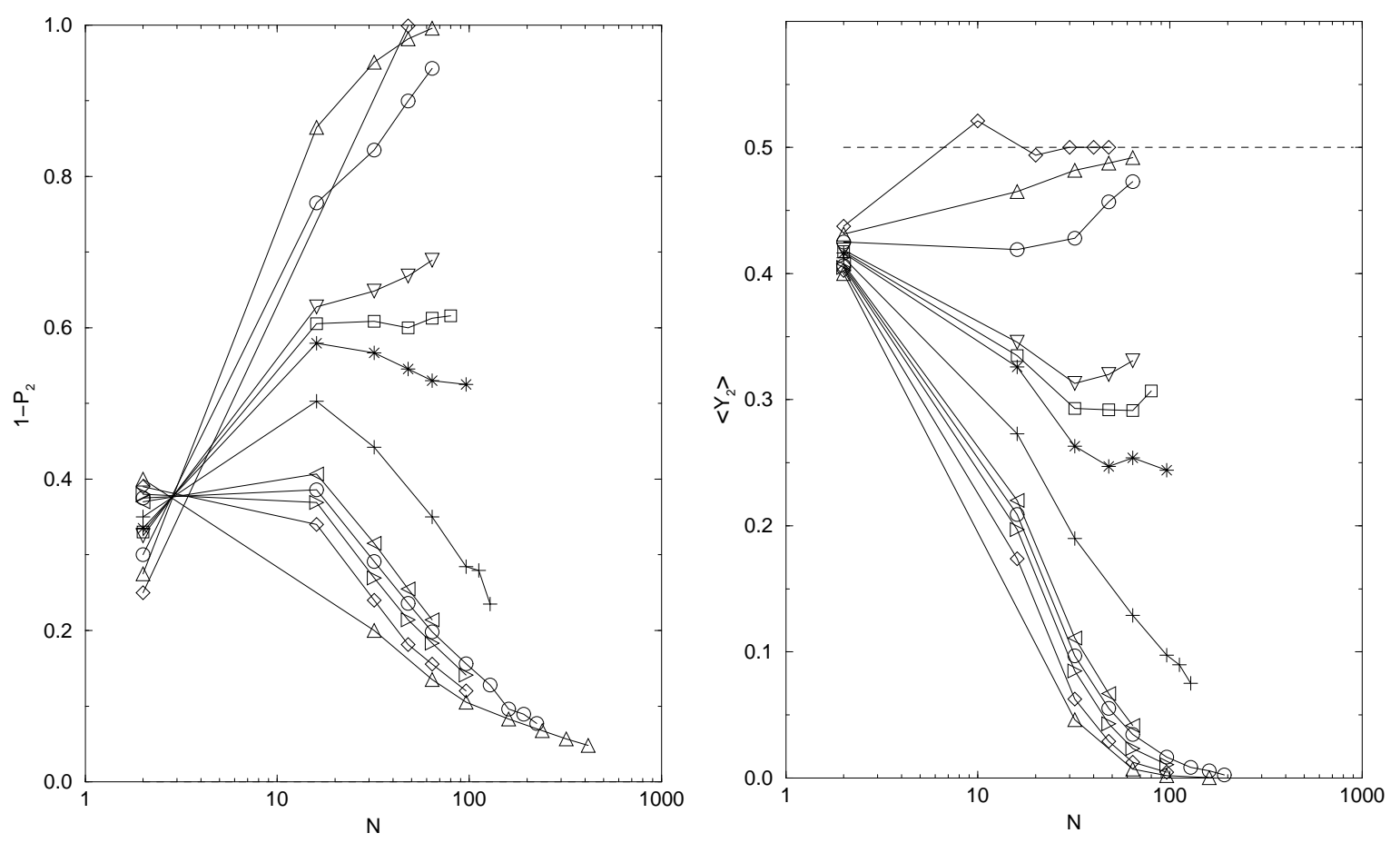

Figure 1: (a): Probability to find a cycle of length different from 2, and (b): Average weight of the attraction basins, as a function system size. From top to bottom, the values of the symmetry is $\eta=0,0.1,0.2,0.3,0.32$ (squares), 0.34 (stars), 0.4, 0.48, 0.5, 0.52, 0.56, 0.6. The error bars are, in the worst cases, comparable with system size.

\subsection{Properties of the attractors}

In order to monitor the transition from short cycles to long attractors, we measured 5 quantities: the probability that a cycle of length 2 is reached, $P(2)$, the average weight of the attraction basins $\overline{Y_{2}}$ (equation 14), the average length of the cycles and of the transient time, and the distribution of cycle length.

The probability to reach a cycle of length $2, P_{2}$, initially decreases with system size. For $\eta \geq 0.34$, it reaches a minimum and starts to increase, going asymptotically to the value 1 . For $\eta \leq 0.32$ it seems that it always decreases with system size, apparently going to zero (though we can not exclude that it starts to increase at a size larger than the ones that we could simulate). Thus we place the transition at $\eta_{c} \approx 0.33$ (see Fig. 19a), remarking that the threshold could be overestimated.

The average weight of the attraction basins has also a non-monotonic behavior with system size, but it leads to a different systematic error: it starts decreasing with system size, then reaches a minimum and eventually increases for $\eta \leq 0.32$, probably tending to the value 0.5 typical of a Random Map with reversal symmetry (dashed line in Fig. 1 b). For $\eta \geq 0.34 \overline{Y_{2}}$ apparently tends to vanish, although we can not exclude that it starts to increase at a larger size. Thus also from this measure we estimate $\eta_{c} \approx 0.33$, but this time the threshold could be underestimated. From this measurement and the previous one, we conclude then that $\eta_{c}=0.33 \pm 0.01$.

We fitted the average length of the cycles and the average transient time with an expo- 


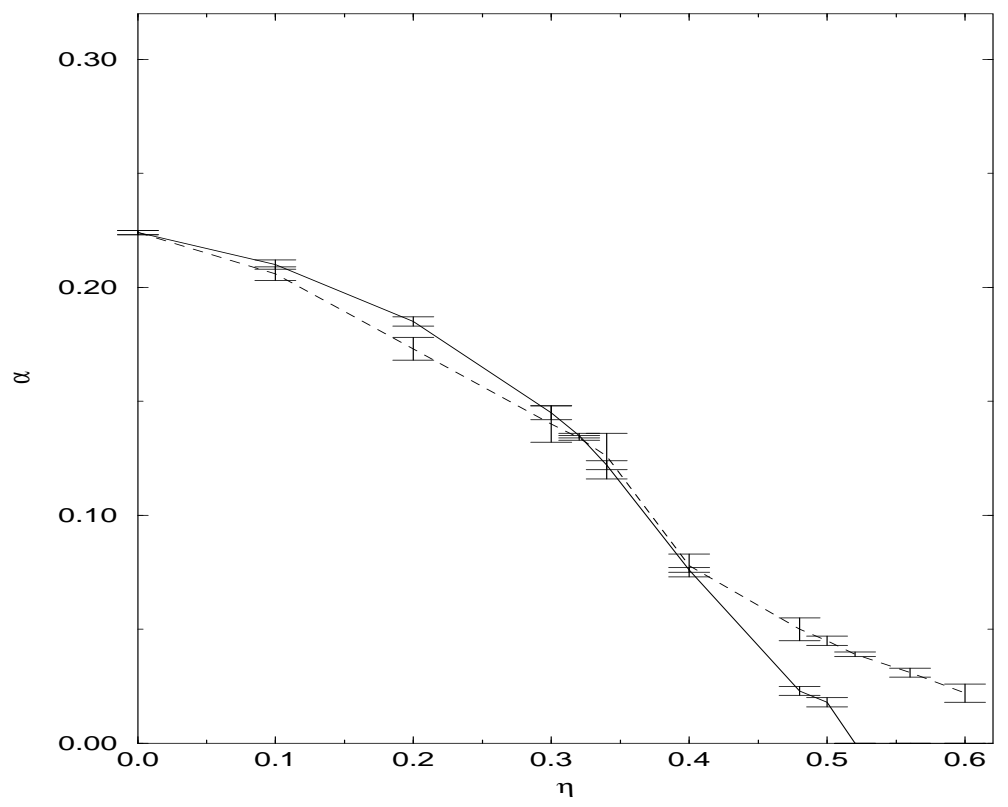

Figure 2: Exponent of the average cycle length (full line) and of the average transient time (dashed line) as a function of the asymmetry.

nential function of system size, $L \simeq \exp \left(\alpha_{L}(\eta) N\right)$ and $T \simeq \exp \left(\alpha_{T}(\eta) N\right)$ respectively. In the first case, the fit is good for $\eta \leq 0.5$, and the exponent $\alpha_{L}(\eta)$ vanishes at this point. For larger symmetry, the average length of the cycles tends to 2 , but not in a monotonic way: it starts increasing, reaches a maximum and then decreases. Thus we can not exclude that the exponential increase of the cycles stops at a smaller value of the symmetry parameter if larger systems are considered.

The average transient time increases as $\exp \left(N \alpha_{T}(\eta)\right)$ for $|\eta|<1$, and $\alpha_{T}(\eta)$ vanishes at $\eta=1$ as $(1-\eta)^{2.0 \pm 0.1}$, as expected. At $\eta=1$ the transients grow as a power law of system size, $\operatorname{Tr} \propto N^{b}$, with $b=0.66 \pm 0.01$, in agreement with the prediction of equation 12 and the previous numerical work by Nützel [10]. At $\eta=0.9$ we found two regimes: for $N \leq 512$, the transient time increases as a power law with exponent $b=0.66$ as in the system with $\eta=1$. At $\eta>1000$ the transient time increases faster with $N$. The analysis of the closing probability, which is a less noisy measure, allow one to conclude that the behavior with $N$ is, asymptotically, exponential with a very small exponent. The first regime reflects the time needed to reach the asymptotic distribution of the overlap, as discussed in section 2 for the case $\eta=1$, as predicted in the previous section.

We plot in Fig. 22 the exponents $\alpha_{L}(\eta)$ and $\alpha_{T}(\eta)$, for $\eta \geq 0$. We checked for $\eta=-0.4$ that, asymptotically in system size, it holds $\alpha_{L}(-\eta) \approx \alpha_{L}(\eta)$ and $\alpha_{T}(-\eta) \approx \alpha_{T}(\eta)$.

The distributions of the lengths of the cycles have different features in three different regions. As a general rule, we have to consider separately cycles of even length and cycles of odd length, not only because the first ones can be obtained in two different ways (they close with $q(t, t+l)=1$ and $q(t, t+l / 2)=-1)$ but also because the closing probability that $q(t, t+l)=1$ is larger for even $l$. That's what we observed: 


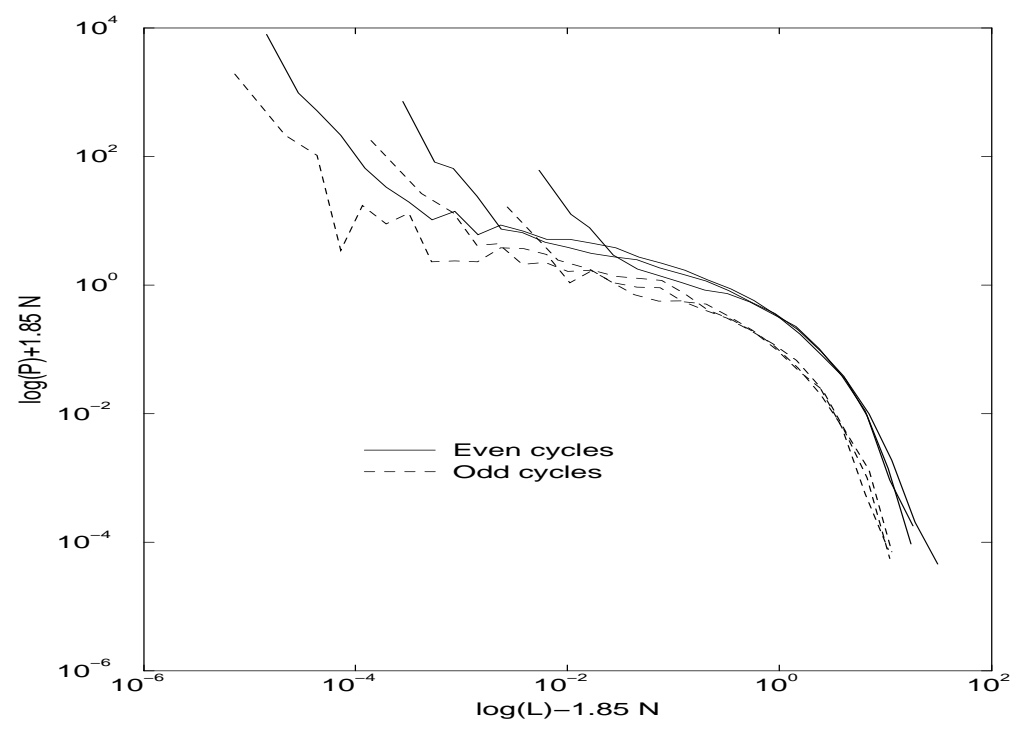

Figure 3: Probability distribution of the rescaled length of the cycles in the chaotic phase $(\eta=0.2) . \quad N=32,48$ and 64 . Systems of different sizes have been rescaled.

1. $\eta \leq \eta_{c}$ : in this region the distribution can be divided in two parts: a power-law tail for short cycles, and an almost exponential tail for long cycles. In this part of the distribution, we found that the rescaled variable $L / \exp \left(\alpha_{L}(\eta) N\right)$ has a well-defined limit distribution when $N$ increases.

As an example, we show in Fig. 3 three systems of different sizes with $\eta=0.20$.

2. $\eta_{c}<\eta \leq 0.5$ : in this region, the distribution is a power-law with exponent smaller than 2. The average value is dominated by the tail of the distribution. The scaling with the average period does not hold. As an example, we show three systems of different sizes with $\eta=0.48$ (figure 国a).

3. $\eta>0.5$ : in this case, the average period tends to 2 . The distributions for the even cycles have approximately the shape of a power-law with an exponent larger than 2 and increasing with system size. The distribution of cycles of odd length is also approximately a power-law, with an exponent that does not decrease with system size, but its total weight goes to zero as $N \rightarrow \infty$. As an example, we show in Fig. 㳑 four systems with $\eta=0.60$.

\subsection{Relaxation of the energy}

The function $E(t)=N^{-1} \sum_{i}\left|h_{i}(t)\right|$, where $h_{i}(t)$ is the local field experienced by neuron $i$ at time $t$, is a Ljapunov function for the symmetric system $(\eta=1)$. In asymmetric networks, its average value is still a non-increasing function of time, even if it may increase in some realizations. 

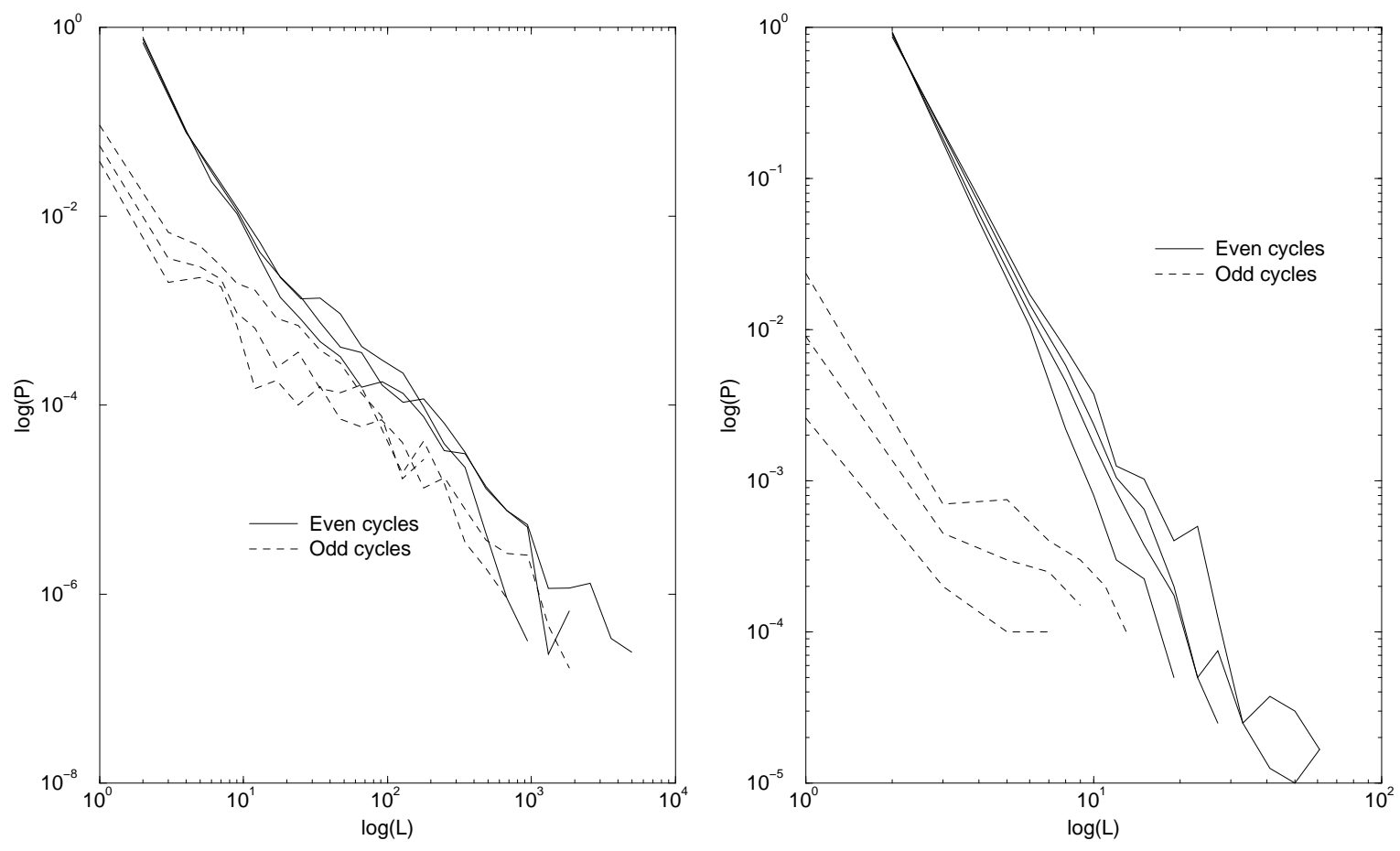

Figure 4: Probability distribution of the length of the cycles in the oscillatory phase: (a) $\eta=0.48$, (b) $\eta=0.60$. Odd and even lengths are shown separately. $N=32,48$ and 64 (from top to bottom).

We show in Fig. 5a $\overline{E(t)}$ for $N=256$ and several values of $\eta$ between 0 and 1 . We imposed the condition that the trajectory is not yet closed when the energy is measured. Without this condition, at high symmetry the trajectories ultimately find cycles of length 2 and the energy reaches a stationary value corresponding to the average energy of such cycles. With the opening condition the energy decreases to lower values. The effect of the opening condition is very small (not even significant) at small $\eta$ and increases as $\eta$ grows.

At $\eta=0$ the energy density is constant in time, and is equal to the expectation value of the module of a Gaussian variable, $E(\eta=0)=\sqrt{2 / \pi}=0.798$. At larger $\eta$, the asymptotic value of the energy is lower and is attained later in time. In Fig. 5 we show the relaxation of the energy for different symmetries, for systems of size $N=256$.

At high symmetry, we observed an interesting phenomenon: the average energy of the fixed points, that are reached with vanishing probability as $N \rightarrow \infty$, is significantly lower than the energy of the typical cycles of length 2. In Fig. fla we show, as a function of $\eta$, the infinite size extrapolation of the energy in cycles of length 1 and of length 2 respectively (the extrapolation was made using $N^{-1 / 2}$ as finite size scaling, which gives very good fits). It can be seen that the difference increases with $\eta$. At $\eta=1$ the energy of cycles of length 2 , which is also the typical energy of the parallel dynamics, is slightly higher than the one computed in 29] with a Monte Carlo simulation of the infinite size dynamics. The energy of the fixed point, on the other hand, is much lower, and its extrapolated value, $-1.55 \pm .01$, could be even lower than the zero temperature energy of the SK model, $E_{0}=-1.526$. This difference seems to reflect a more general tendency: the cycles whose length is odd have a lower energy than cycles of even length (figure 6 6b). This effect can be due to the fact that 


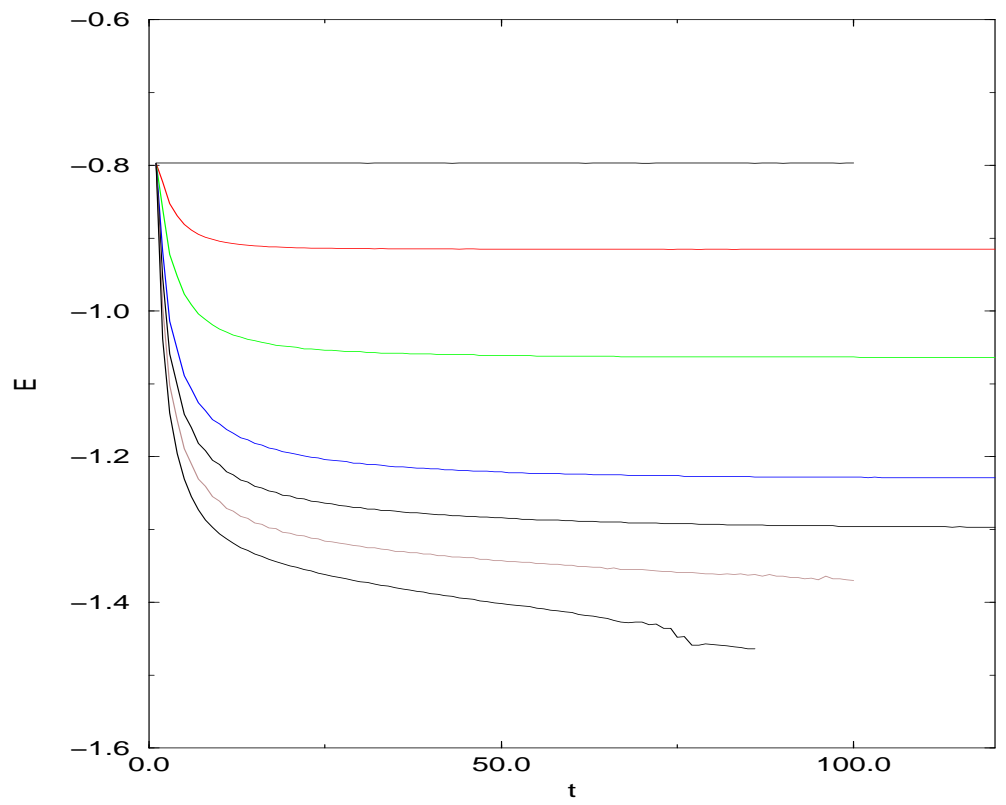

Figure 5: $\overline{E(t)}$ on trajectories not yet closed for $N=256$ and $\eta$ respectively equal to $0,0.32$, $0.50,0.60,0.80,0.90$ and 1 . The energy is a decreasing function of $\eta$.

cycles of even length are more rapidly found than cycles of odd length (the typical transient times are shorter, as we expect from the closing probabilities), so the energy has more time to decrease.

\subsection{Overlap and closing probabilities}

Though it is not the main point of this work, we present here some numerical results about the distribution of the overlap, measured on trajectories not yet closed (opening condition). All our data in this section refer exclusively to the distribution of $q(t, t+l)$ subjected to such a condition.

The first figure that we show refers to the mean value and to the variance of the distribution of $q(t, t+l)$. We show these quantities as a function of $t$ for different values of $l$. As we noted in section 2 , the average overlap is zero due to symmetry when the time difference $l$ is odd, so we show its value only for even $l$. For $\eta>0$, it is always a non-decreasing function of $t$, and it reaches soon an asymptotic value (figure $7 \mathrm{a}$ ). The fact that $Q(t, t+l)$ is non-decreasing means that it is more and more difficult to lose the memory of the configuration as time increases. The asymptotic value, $Q_{l}^{*}$, is a decreasing function of $l$. We show its relaxation for different values of the asymmetry in Fig. 8a. It is evident from the figure that the relaxation becomes slower and slower as $\eta$ increases. Pfenning, Rieger and Schreckenberg [9] and Eissfeller and Opper [12] observed a transition at $\eta=0.825$ between a regime at high symmetry where $Q(0, l)$ relaxes as a power-law to a non-vanishing limit value, $Q(0, l) \approx Q_{\infty}+A l^{-a}$, and a regime at low symmetry where the relaxation is exponential and the remanent magnetization vanishes, $Q(0, l) \propto \exp (-a l)$. We expect to observe the same transition for the asymptotic value of $Q(t, t+l)$ 円, but our data do not allow us to verify this

\footnotetext{
5 We note that, due to the opening condition, this quantity has a meaning different from the usual
} 

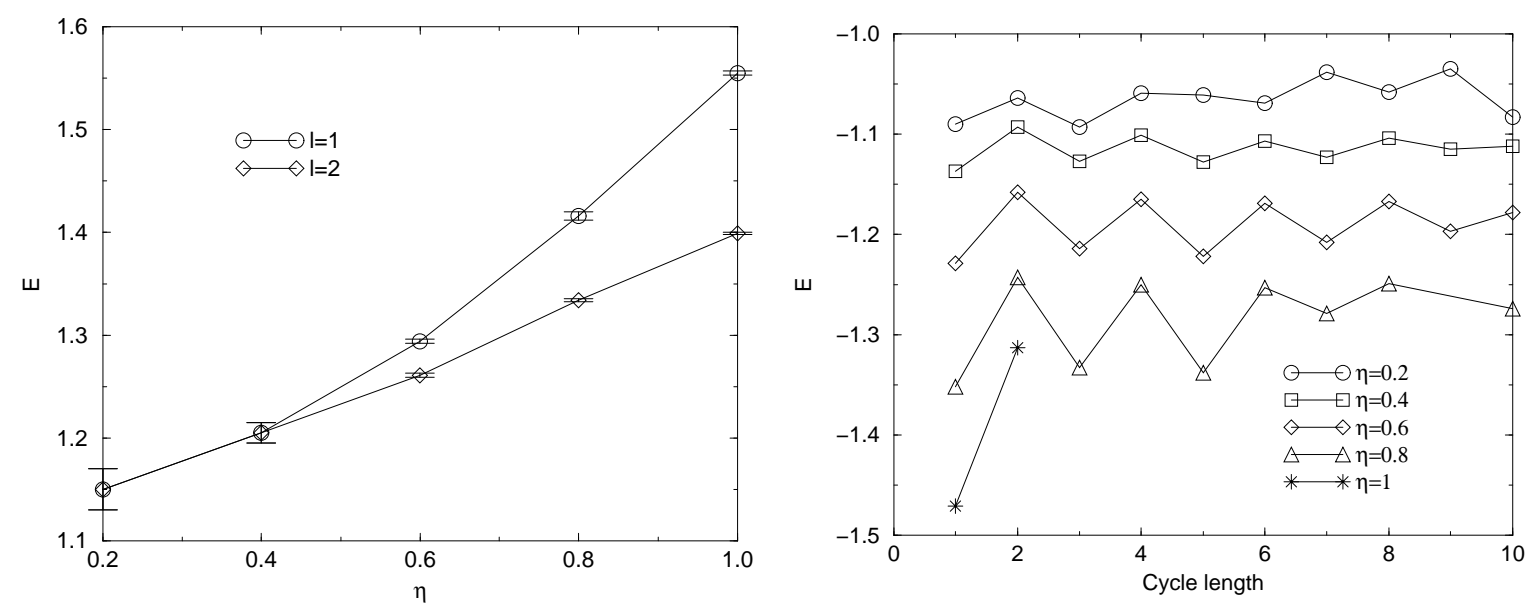

Figure 6: (a) $\bar{E}$ in cycles of length 1 and 2, as a function of $\eta$. The data are obtained extrapolating to the infinite size through the fit $E_{N}=E_{\infty}+A N^{-1 / 2}$. (b) $\bar{E}$ as a function of cycle length $l$ for systems with $N=64$ and different symmetries. For large symmetry there are no data for odd $l$, (except $l=1$ ) since such cycles are met very rarely. For small symmetry the small cycles are almost never found, and the error is very large.

point.

Figure $7 \mathrm{~b}$ shows the variance of $q(t, t+l)$ as a function of $t$ for different values of $l$. The variance is always an increasing function of $t$, and reaches an asymptotic value as $t$ increases. The cases of odd $l$ and even $l$ have to be distinguished. The variance is larger for $l=2 m+1$, and decreases as a function of $m$. For $l=2 m$ the variance is smaller, and increases as a function of $m$. The asymptotic value of $V(t, t+l)$ is shown as a function of $l$ (for even and odd $l$ separately) in Fig. 8b Since odd and even variances have an opposite behavior as a function of $l$, the function $\tilde{V}(t ; l)=(V(t, t+l)+V(t, t+l+1)) / 2(l$ odd) shows a very small dependence on $l$. This dependence is however systematic: $\tilde{V}(t ; l)$ is an increasing function of $l$ when $t$ is large, and decreasing when $t$ is small. In particular, $\tilde{V}(0 ; l) \approx 1$ (the totally random case) for every value of $\eta$. We also verified that, for $\eta \simeq 1$ and $t$ large, it holds $V(t, t+2) \propto 1-Q(t, t+2)$, relation that we used in section 2 .

The closing probability $\pi_{N}(t, t+2)$ also increases as a function of $t$ to an asymptotic value $\pi_{N}^{*}(2 ; \eta) \propto e^{-N \alpha_{2}(\eta)}$. We plot in Fig. 9a the behavior of $\left(\pi_{N}^{*}(2 ; \eta)\right)^{1 / N}$ as a function of $\eta$ for systems of different sizes. It appears that it converges very slowly to a function independent of $N$, which is an even function of $\eta$, has a cusp in $\eta=0$ and is concave downward. The limit value for large $N$ is 1 for $\eta=1$, because $\pi_{N}^{*}(2 ; \eta=1)$ decreases as a power-law of $N$, and is less than 1 for $\eta<1$, indicating that the exponential scaling is fulfilled for $\eta<1$.

Before going deeper inside the analysis of the closing probabilities, we note that all the quantities that we observed, say $Q_{N}(t, t+l), V_{N}(t, t+l)$ and $\pi_{N}(t, t+l)$, reach only approximately an asymptotic value in $t$. In reality, all these quantities must decrease with $t$, at least for time scales of the order of the inverse of the closing probability (see section 2 ). This is observed in the simulations. However, the discussion presented above, based on stationary closing probabilities, is not modified essentially by this fact, since the decrease of

Edwards-Anderson order parameter, which measures the size of an asymptotic state of the dynamics. 

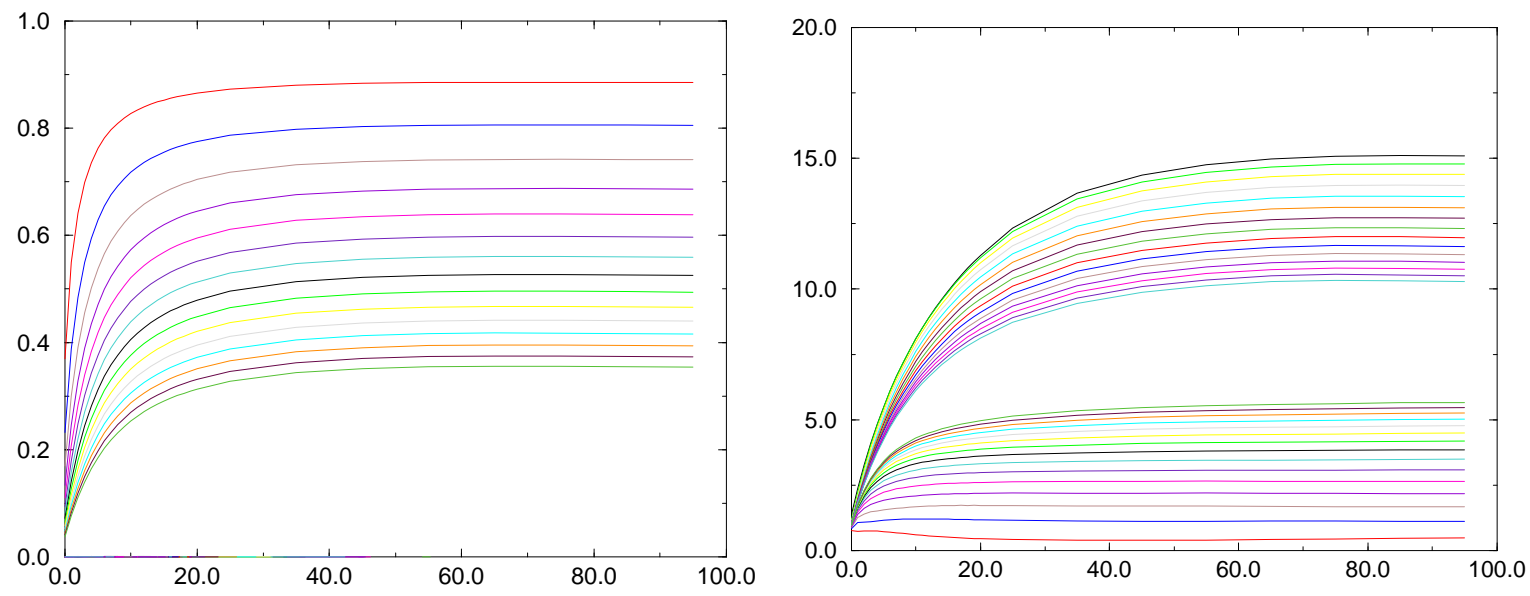

Figure 7: Average value $Q(t, t+l)$ (a) and variance $N V(t, t+l)$ (b) of the overlap $q(t, t+l)$ over trajectories not yet closed. Here $\eta=0.6$ and $N=128$. The different curves are for different values of $l$. In the first figure, only even values of $l$ between 2 and 30 are shown, from top to bottom. In the second figure the lower bundle of curves corresponds to even values of $l$ (and $V(t, t+l)$ is an increasing function of $l$ ), the higher bundle corresponds to odd $l$ (and $V(t, t+l)$ is a decreasing function of $l$ ).
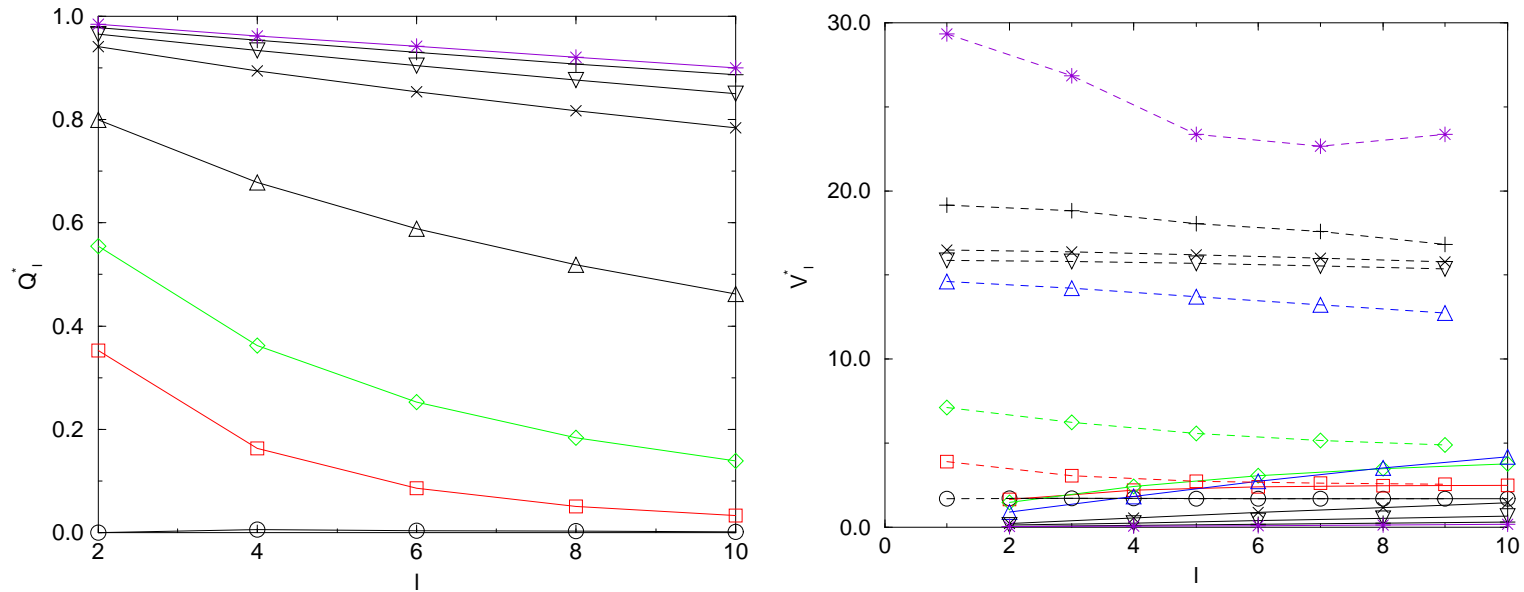

Figure 8: Large time value of $Q(t, t+l)$ (a) and $N V(t, t+l)$ (b) as a function of $l$ for $N=192$ and $\eta=0,0.2,0.32,0.5,0.7,0.8,0.9,1$. In the first figure only even values of $l$ are shown and $\eta$ grows from bottom to top. In the second figure even values of $l$ are represented as a dashed line ( $\eta$ grows from bottom to top) and odd values as a solid one ( $\eta$ grows from top to bottom for $l=2$ ). At $\eta=0$ there is no difference between even and odd $l$. 


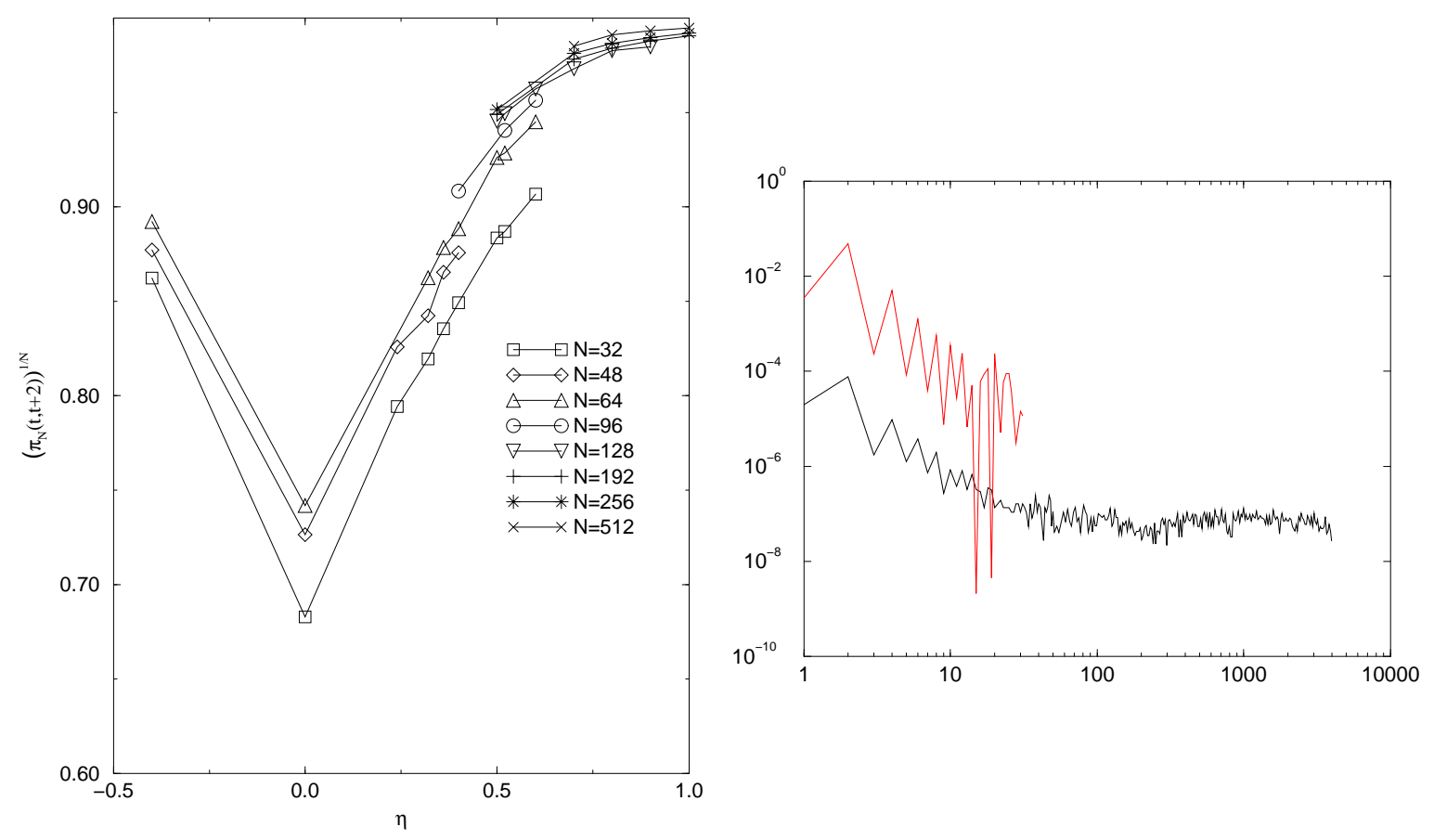

Figure 9: Closing probability for cycles of length 2 as a function of $\eta$ for systems of different sizes (a) and as a function of cycle length $l$, for $N=64$ and $\eta=0.32$ and 0.56 (from bottom to top).

the closing probability becomes slower and slower when system size increases: we find, for very large $t$, and for $l=2, \pi_{N}(t, t+2) \propto t^{-b_{N}}$, with $b_{N} \propto N^{-(1+\epsilon)}$. Thus $\pi_{N}(t, t+l)$ can be considered constant even on time scales exponentially increasing with $N$, like the ones involved in the closure of the cycles.

We then report the asymptotic closing probability $\pi_{N}^{*}(l ; \eta)$ as a function of $l$ for two different values of $\eta$. The characteristic oscillations for even-odd $l$ show up. Apart for that, the closing probability is a decreasing function of $l$. It is not clear whether it reaches a stationary value, $\exp \left(-N \alpha_{\infty}(\eta)\right)$, when $l$ increases. It is very difficult to measure the closing probability for large $l$. In any case, we find that, contrarily to our hypothesis, the value of $l$ at which $\pi_{N}^{*}(l ; \eta)$ seems to become stationary increases with $N$. Thus the exponent $\alpha_{\infty}(\eta)$ seems not to exist, and in any case it can not be determined numerically.

\section{Discussion}

It is known that the deterministic dynamics of asymmetric neural networks exhibits complex features. Among these features, we investigated here the transition between cycles of length 2 and "chaotic" (exponentially long) attractors, first reported in [10]. We found out that, though the average cycle length shows an abrupt change at $\eta_{L}=0.5$, the region where cycles of length 2 are stable extends up to $\eta_{c}=0.33 \pm 0.01$. In this region nearly all the trajectories end up in cycles of length 2. The number of these trajectories is exponentially high and the weight of their attraction basins is vanishingly small. At lower symmetries the probability to find a cycle of length 2 drops abruptly to zero (though their number is still exponentially 
high). The typical attractors are now cycles exponentially long with system size, whose number increases only proportionally to $N$. The weights of the attraction basins seem to have the same distribution as in a Random Map model with reversal symmetry [24, 21]. The typical times after which attractors are reached, on the other hand, vary exponentially with system size for every finite asymmetry, and vary as a power-law, $\operatorname{Tr} \propto N^{2 / 3}$, for $|\eta|=1$.

All these features can be predicted with arguments relying, through the closing probabilities, on the distribution of the overlap in an infinite system, but the point where the transition takes place can not be computed without a more detailed knowledge of the overlap distribution. However, this transition does not correspond to a transition in the infinite system. Asymmetric neural networks do present such a transition from a phase at low symmetry where the remanent magnetization vanishes, $Q_{\infty}=\lim _{l \rightarrow \infty} Q(0, l)=0$ (loss of memory) and a phase at high symmetry where the remanent magnetization is finite. This takes place at $\eta_{R M}=0.825$ [9, 12], which is a value much larger than $\eta_{c}=0.33$, at which cycles of length 2 cease to be the typical attractors.

We can explain qualitatively the inclination of the trajectories to close on cycles of length 2 as a consequence of the parallel updating and of the symmetry of the interaction. Both these elements conspire to create an effective ferromagnetic interaction between the spin $\sigma_{i}(t)$ and the same spin two time steps later (for negative $\eta$ the effective interaction is antiferromagnetic, and it tends to reverse the spins after two time steps, thus resulting in cycles of length 4). The transition takes place when the sum of the closing probabilities of long cycles balances the one of cycles of length 2. This balance involves both the number of cycles and the weights of their attraction basins. Cycles of length 2 are much more than cycles of any other length at every value of $\eta>0$ (their number grows exponentially with system size with an exponent which is the double of the same exponent for cycles of length 1, [5]), but their attraction basins are vanishingly small, and, at a certain point, very long cycles, whose number grows only linearly with system size, represent the overwhelming majority of phase space.

The dynamics of the system is a kind of relaxation, the function $E(t)$ defined in (3) playing the role of an "energy". This analogy is exact in the symmetric system, where $E(t)$ decreases at every time step until a cycle is reached. In the asymmetric systems $E(t)$ decreases in average, but not in every realization. The asymmetry introduces something similar to thermal noise in the dynamics: the average asymptotic value of $E$ increases when decreasing $\eta$. Fixed points are states of low energy, but they are very difficult to reach because of the competition of higher energy attractors (either cycles of length 2 or very long cycles), which are easier to reach.

We can ask ourselves what changes in the above description if thermal noise is introduced. Of course thermal noise destroys the limit cycles which are produced by the deterministic dynamics, but at low temperature some metastable states reminiscent of the cycles of length 2 may still survive. This discussion may be put on precise basis in symmetric networks with $\eta=1$. In this case detailed balance is fulfilled with a suitable definition of the noise, and at equilibrium the statistical state of the system is described by a kind of Boltzmann distribution, for which the fluctuation-dissipation theorem holds: 


$$
\operatorname{Pr}\left(\sigma_{1} \cdots \sigma_{N}\right) \propto \prod_{i=1}^{N}\left(e^{\beta h_{i}}+e^{-\beta h_{i}}\right),
$$

where $h_{i}=\sum_{j} J_{i j} \sigma_{j}$ is the local field experienced by spin $i$ [19]. This statistical description does not hold anymore for asymmetric networks, for which detailed balance breaks down.

Ferraro [28] and Scharnagl et al. [29] computed the asymptotic value of the energy, which in this model is defined as $\left\langle h_{i} \tanh \left(\beta h_{i}\right)\right\rangle$, and at $\beta=\infty$ coincides with definition 3, for the system with $\eta=1$. They used the Monte-Carlo scheme of [17], which gives results free of finite size effects (even if for computational reasons there is a limit of $t \approx 100$ on the time steps that can be reliably performed). At temperatures larger than 0.6 the energy follows the theoretical prediction for the SK model with remarkable accuracy, as expected in [30], but at lower temperatures the energy is considerably higher than what expected for the SK model [28, 29]. This may due to the fact that the system remains trapped in metastable states corresponding to energy minima that at $T=0$ are cycles of length 2 . As we observed, the energy of these states is significantly higher than the energy of the fixed points (which are low energy states for the SK model) and their attraction basins cover nearly all of phase space. We did not do direct simulations to test this interpretation, nor to see whether these states are also found for asymmetric couplings up to some critical value of the asymmetry, but we think that this could be an interesting issue.

Asymmetric networks at finite temperature were recently simulated in [14, though with Langevin dynamics whose equilibrium distribution, for $\eta=1$, is given by the SK model. The authors found aging effects and non-trivial overlap distributions at small but finite asymmetry. We agree with them about the absence of aging for $\eta<0.8$ : for these systems, the average overlap $Q(t, t+l)$, subject to the opening conditions, reaches for every $l$ a timetranslation-invariant state, where it does not depend anymore on $t$. Our data suggest that this could not hold anymore at larger values of $\eta$ (and it can be speculated that the threshold coincides with $\eta_{R M}=0.825$ at which, where the remanent magnetization is different from zero), but the time-window that we analyzed is to small to state anything definite about this point.

The results that we present here have also to be compared with a recent investigation of the relaxation dynamics of the SK model at $T=0$ [31. Using different kinds of dynamics, defined as reluctant, sequential and greedy, it was found, among other things, that the typical fixed points reached have different energy densities for the different dynamics $\left(E_{g}=-1.416\right.$, $E_{S}=-1.430$ and $\left.E_{r}=-1.492\right)$. The energy density that we found for $\eta=1$, extrapolated to the infinite size, is higher in cycles of length $2\left(E_{2}=-1.399\right)$, but it is lowest on the fixed points $\left(E_{1}=-1.55 \pm .01\right)$, and it could be even lower than the zero-temperature energy of the SK model $E_{0}=-1.526$.

The transition that we investigated presents some features similar to the one taking place in Kauffman networks, a disordered dynamical system proposed as a model of genetic regulation [16]. Also in that case the average length of the attractors increases exponentially in the chaotic phase, where the weights of the attraction basins follow the Random Map distribution 22, and do not depend on system size in the frozen phase. Nevertheless, despite the similarity between the chaotic phases of the two models, the frozen phases are quite different. In the frozen phase of Kauffman model the number of attractors has a 
finite limit as system size increases, the average weight of the attractors does not vanish and the transient time is also finite. Moreover, in the infinite size limit a phase transition corresponding to the transition for the attractors takes place, between a phase without damage spreading and a phase where a small damage propagates to the whole system [32]. None of these features are present in asymmetric neural networks. While the transition in Kauffman networks is a consequence of its finite connectivity (every element receives inputs from exactly $K$ elements), which in turn implies that, in the frozen phase, only a finite number of elements are relevant for the dynamics [33, 34], asymmetric neural networks are a system with infinite connectivity, and their oscillatory phase shows much less order than the frozen phase of Kauffman networks.

Though being a system with a finite number of states, this system shows for $\eta=0$ and in the infinite size some relevant features of chaos in continuous systems [13]. At $\eta=1$, on the other hand, the system never loses memory of its initial condition. At intermediate symmetries the system shows features that are "chaotic" and features that are "ordered". The analogy can be carried out also through the study of the attractors. In discretized chaotic systems "artificial" limit cycles are present due to the finiteness of phase space. The attraction basins of these cycles follow a Random Map statistics [27], which is in agreement to what is observed here for $\eta<0.33$. The closing time of these cycles increases as $\epsilon^{-D_{2} / 2}$ [35, 36, 27], where $\epsilon$ is the discretization and $D_{2}$ is the correlation dimension [37. This is also analogous to what is observed in the present model for $\eta<0.33$ if we identify $2^{-N}$ with $\epsilon^{D}$ and $\alpha_{\infty} / \ln 2$ with $D_{2} / D$ (the fact that, even in the most "chaotic case" $\eta=0$, we find $\alpha_{\infty}<\ln 2$ can be interpreted as if in this model the correlation dimension were always less than the dimension $D$ of the embedding space). But for $\eta>0.33$ the situation is less clear: still the closing time increases exponentially with system size, as if there were a finite "correlation dimension" of the asymptotic configurations equal to $\alpha_{2} / \ln 2$, but the length

of the cycles does not increase with $N$ and the statistic of the attraction basins is not of the Random Map type. Moreover, the number of cycles increases exponentially with $N$. Thus neither the analogy with a discretized chaotic system, nor the analogy with a periodic system hold.

\section{Acknowledgments}

UB is pleased to thank Gerard Barkema, Peter Grassberger, Normann Mousseau, Paolo Muratore Ginanneschi, Heiko Rieger, Felix Ritort and Angelo Vulpiani for interesting discussions.

\section{References}

[1] G. Parisi (1986), Asymmetric neural networks and the process of learning, J. Phys. A: Math. Gen. 19, L675

[2] J. Hertz, G. Grinstein and S. Solla (1987), Neural nets with asymmetric bonds, in Heidelberg Colloquium on Glassy Dynamics, J.J. van Hemmen and I. Morgenstern ed., Spinger-Verlag , Berlin 
[3] A. Crisanti and H. Sompolinsky (1987), Dynamics of spin systems with randomly asymmetric bonds: Langevin dynamics and a spherical model, Phys. Rev. A 364922.

[4] A. Crisanti and H. Sompolinsky (1988), Phys. Rev. A 374865.

[5] H. Gutfreund, J.D. Reger and A.P. Young (1988), The nature of attractors in an asymmetric spin glass with deterministic dynamics, J. Phys. A: Math. Gen. 21, 2775

[6] Spitzner and Kinzel (1989), Z. Phys. B 74527

[7] H. Rieger, M. Schreckenberg, and J. Zittartz (1989), Glauber dynamics of the asymmetric SK model, Z. Phys. B - Condensed Matter 74, 527

[8] H. Rieger, M. Schreckenberg, P. Spitzner and W. Kinzel (1991), Alignment in the fully asymmetric SK model, J. Phys. A: Math. Gen. 24, 3399

[9] T. Pfenning, H. Rieger and M. Schreckenberg (1991), Numerical investigation of the asymmetric SK model with deterministic dynamics, J. Phys. I 1, 323

[10] K. Nützel (1991), The length of attractors in asymmetric random neural networks with deterministic dynamics, J. Phys. A: Math. Gen. 24, L151.

[11] K. Nützel, U. Krey (1993), Subtle dynamical behavior of finite size SherringtonKirkpatrick spin glasses with non symmetric couplings, J. Phys. A: Math. Gen. 26, L591

[12] Eissfeller, Opper (1994), Mean-field Monte Carlo approach to the SherringtonKirkpatrick model with asymmetric couplings Phys. Rev. E 50709

[13] A. Crisanti, M. Falcioni and A. Vulpiani (1993), Transition from regular to complex behavior in a discrete deterministic asymmetric neural network model, J. Phys. A: Math. Gen. 26, 3441

[14] G. Iori, E. Marinari (1996) On the stability of the man-field glass broken phase under non-Hamiltonian perturbations, J. Phys. A: Math. Gen. 30 (1997) 4489

[15] L. Molgedey, J. Schuchhardt and H.G. Schuster (1992), Suppressing chaos in neural networks by noise, Phys. Rev. Lett 69, 3717

[16] S.A. Kauffman (1969), J. Theor. Biol. 22, 437 Homoeostasis and Differentiation in Random Genetic Control Networks, Nature 244, 177-178. S.A. Kauffman (1993), Origins of Order: Self-Organization and Selection in Evolution, Oxford University Press

[17] H. Eissfeller and M. Opper (1992) New method for studying the dynamics of disordered spin systems without finite-size effects, Phys. Rev. Lett. 682094

[18] J.J. Hopfield (1982), Neural networks and physical systems with emergent collective computational abilities, Proc. Nat. Acad. Sci. USA, 79, 2554 
[19] D.J. Amit (1989), Modelling Brain Function, Cambridge University Press; P. Peretto (1984), Biol. Cybern. 50, 51; E. Goles (1986), in Disordered Systems and Biological Organization, E. Bienenstock, F. Fogelman Soulié and G. Weisbuch ed., SpringerVerlag, Berlin.

[20] S. Kirkpatrick and D. Sherrington (1978), Infinite-ranged models of Spin Glasses, Phys. Rev. B 17, 4384

[21] U. Bastolla, G. Parisi (1997) Attractors in fully asymmetric neural networks, Jour Phys A: Math. Gen. 305613.

[22] U. Bastolla and G. Parisi (1966), Closing probabilities in the Kauffman Model: an annealed computation, Physica D 98, 1

[23] E. Gardner, B. Derrida, P. Mottishaw (1987) J. Physique (Paris) 48, 741

[24] B. Derrida, H. Flyvbjerg (1986), The random map model: a disordered model with deterministic dynamics, Journal de Physique 48, 971-978

[25] M. Schreckenberg (1992), Attractors in the fully asymmetric SK model, Z. Phys. B Condensed Matter 86, 453.

[26] B. Derrida and H. Flyvbjerg (1986), Multivalley Structure in Kauffman's Model: Analogy with Spin Glasses, J.Phys.A: Math.Gen. 19, L1003-L1008

[27] U. Bastolla and G. Parisi (1997), Attraction basins in discretized maps, J. Phys. A: Math. Gen., 30, 3757

[28] G. Ferraro (1994) Infinite volume relaxation in the Sherrington-Kirkpatrick model, cond-mat 9407091

[29] A. Scharnagl, M. Opper, W. Kinzel (1995) On the relaxation of the infinite-range spin glass J. Phys. A: Math. Gen. 285721

[30] R. Brunetti, G. Parisi, F. Ritort (1992) Phys. Rev. B 465339

[31] G. Parisi, On the statistical properties of the large-time zero-temperature dynamics of the SK model, cond-mat/9501045

[32] B. Derrida and Y. Pomeau (1986), Random Networks of Automata: a Simple Annealed Approximation, Biophys. Lett. 1(2), 45-49

[33] H. Flyvbjerg (1988), An order parameter for networks of automata, J. Phys. A: Math. Gen. 21 L955

[34] U. Bastolla, G. Parisi (1997), Relevant elements, magnetization and dynamical properties in Kauffman networks: a numerical study, Physica D, to appear, and cond-mat 9708213

[35] C. Grebogi, E. Ott, J.A. Yorke (1988) Roundoff-induced periodicity and the correlation dimension of chaotic attractors, Phys. Rev. A 383688 
[36] C. Beck (1989) Scaling behavior of random maps, Phys. Lett. A 136121

[37] P. Grassberger, I. Procaccia (1983) Characterization of strange attractors Phys. Rev. Lett. 50346 